\title{
Attacked from all sides
}

Focal adhesion kinase (FAK, also known as $\underline{\mathrm{PTK} 2}$ ) is a non-receptor protein tyrosine kinase that is overexpressed in many tumours, including ovarian cancer, in which it is predictive of poor clinical outcome. FAK is also overexpressed in tumour-associated endothelial cells, so it is an attractive option for concomitant targeting of tumour cells and the microenvironment. Anil Sood and colleagues now show that a small molecule FAK inhibitor, TAE226, is effective in ovarian cancer mouse models, even those that are resistant to the taxane docetaxel.

Female nude mice bearing HeyA8 ovarian tumours in the peritoneal cavity received either saline as a control, daily oral TAE226, weekly intraperitoneal docetaxel, or both TAE226 and docetaxel. There was a 54-79\% reduction in tumour burden with either TAE226 or docetaxel, and an $89 \%$ reduction with both drugs. When a HeyA8 cell line that is resistant to several chemotherapy agents, including taxanes, was used in the same model, TAE226 alone showed a $46 \%$ reduction in tumour growth and docetaxel alone was not effective, whereas the combination of TAE226 and docetaxel gave an $85 \%$ reduction. In addition, the combination of TAE226 and docetaxel reduced metastases and increased the survival times of the mice. Remarkably, when the combination therapy was started 2 weeks after ovarian tumours were established, it still caused $60 \%$ tumour regression.

So, what are the mechanisms involved? TAE226 reduced levels of phosphorylated FAK, as expected. The FAK inhibitor also decreased tumour cell proliferation, microvessel density, and expression of vascular endothelial growth factor (VEGF) and matrix metalloproteinase 9 (suggesting suppression of angiogenesis), and also caused apoptosis of endothelial cells. Combination therapy had a greater effect than either drug alone. As FAK is known to have a role in the migration of vascular smooth muscle cells, the authors also investigated whether TAE226 affected mature tumour vasculature. They found that TAE226 reduced pericyte coverage of mature vessels in the mouse tumours. Pericytes are thought to protect tumour-associated endothelial cells by producing VEGF locally when stimulated: TAE226 blocked the production of VEGF by pericyte-like 10T1/2 cells despite stimulation.

These studies indicate that FAK is an important therapeutic target in ovarian cancer, and inhibition of its kinase activity is a promising approach. Ezzie Hutchinson

ORIGINAL RESEARCH PAPER Halder, J. et al. Therapeutic efficacy of a novel focal adhesion kinase inhibitor TAE226 in ovarian carcinoma. Cancer Res. 67, 10976-10983 (2007)

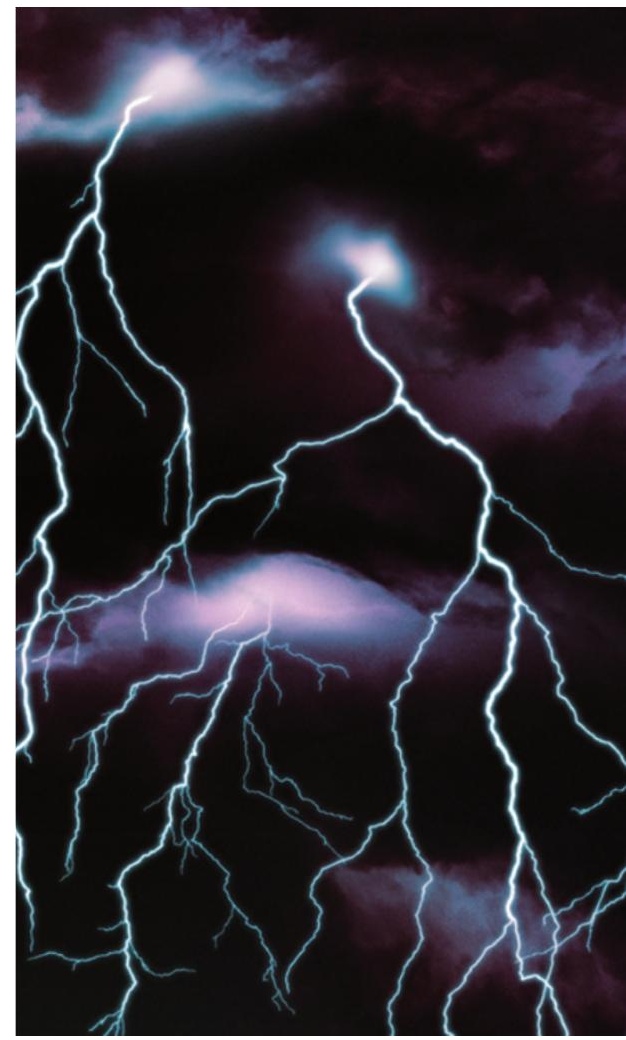

